\title{
NUMBER OF RATIONAL POINTS OF A SINGULAR CURVE
}

\author{
W. A. ZÚÑ̃IGA GALINDO
}

(Communicated by William W. Adams)

\begin{abstract}
In this paper, we give a bound for the number of rational points of a complete, geometrically irreducible, algebraic curve defined over a finite field. We compare it with other known bounds and discuss its sharpness. We also show that the asymptotic Drinfeld-Vladut bound can be generalized to the case of singular curves.
\end{abstract}

\section{INTRODUCTION}

Let $X$ be a complete, geometrically irreducible, algebraic curve defined over a finite field $k=\mathbb{F}_{q}$ with $q$ elements. We denote by $\pi: \widetilde{X} \longrightarrow X$ the morphism of normalization of $X$, by $g$ its arithmetical genus and by $\tilde{g}$ its geometric genus. The curve $X \times_{k} \bar{k}$ is denoted by $\bar{X}$, where $\bar{k}$ is the algebraic closure of $k$.

We define

$$
\delta:=\sum_{P \in \bar{X}_{\text {sing }}} \delta_{P},
$$

where $\delta_{P}$ is the local degree of singularity at $\mathrm{P}$ (see sect. 2), and

$$
\gamma:=\sum_{P \in \bar{X}_{\text {sing }}} \gamma_{P}
$$

where $\gamma_{P}$ is the dimension of an algebraic group attached to each singular point (see sect. 2). If $X$ is non-singular, Weil's theorem states that the number of rational points on $\mathbb{F}_{q^{n}}$ of $X$ satisfies the inequality

$$
\left|\# X\left(\mathbb{F}_{q^{n}}\right)-\left(q^{n}+1\right)\right| \leq 2 \tilde{g} q^{\frac{n}{2}} .
$$

If $X$ is singular, Serre (cf. [Ser2]) has shown that the number of rational points of $X$ satisfies the inequality

$$
\left|\# X\left(\mathbb{F}_{q}\right)-(q+1)\right| \leq 2 g \sqrt{q} .
$$

Stöhr has found a bound in terms of the difference $\sigma(X)-\sigma(\tilde{X})$, where $\sigma(X)$ and $\sigma(\tilde{X})$ denote the Hasse-Witt invariant of $X$ and $\tilde{X}$ respectively (cf. [Sto], prop. 4.4). More precisely,

$$
\left|\# \tilde{X}\left(\mathbb{F}_{q^{n}}\right)-\# X\left(\mathbb{F}_{q^{n}}\right)\right| \leq \sigma(X)-\sigma(\tilde{X}) .
$$

Received by the editors October 17, 1995 and, in revised form, January 31, 1997.

1991 Mathematics Subject Classification. Primary 11G20; Secondary 14H25.

The author thanks Prof. Karl-Otto Stöhr for many helpful discussions, and the referee for his or her useful comments. Supported by CNPq-Brazil and COLCIENCIAS-Colombia.

(C)1998 American Mathematical Society 
Corollary 4.2 of [Sto] and Weil's bound (1.1) imply

$$
\left|\# X\left(\mathbb{F}_{q^{n}}\right)-\left(q^{n}+1\right)\right| \leq 2 \tilde{g} q^{\frac{n}{2}}+\delta .
$$

Aubry and Perret (cf. [A-P]) also proved (1.3). Our result is the following.

Theorem 3.1. Let $X$ be a complete, geometrically irreducible, algebraic curve defined over a finite field $k=\mathbb{F}_{q}$. Then the number of rational points on $\mathbb{F}_{q^{n}}$ satisfies

$$
\left|\# X\left(\mathbb{F}_{q^{n}}\right)-\left(q^{n}+1\right)\right| \leq 2 \tilde{g} q^{\frac{n}{2}}+\delta-\gamma .
$$

The above bound improves (1.3) and cannot be improve further without additional hypothesis about the singularities of the curve (see example 3.2).

Drinfeld and Vladut have found an asymptotic bound for the number of rational points of a non-singular curve (c.f $[\mathrm{D}-\mathrm{V}])$. More precisely,

$$
\tilde{A}(q)=\limsup _{\tilde{g} \rightarrow \infty} \frac{N_{q}(\tilde{g})}{\tilde{g}} \leq \sqrt{q}-1,
$$

where $N_{q}(\tilde{g})=\operatorname{Max}\left\{\# X\left(\mathbb{F}_{q}\right) \mid X\right\}$ and $X$ runs through all complete, geometrically irreducible, non-singular, algebraic curves defined over $\mathbb{F}_{q}$ of genus $\tilde{g}$.

Analogously, we define

$$
\mathcal{N}_{q}(g)=\operatorname{Max}\left\{\# X\left(\mathbb{F}_{q}\right) \mid X\right\},
$$

where $X$ runs through all complete, geometrically irreducible, algebraic curves defined over $\mathbb{F}_{q}$ of arithmetical genus $g$, and prove

\section{Theorem 4.1.}

$$
\mathcal{A}(q):=\limsup _{g \rightarrow \infty} \frac{N_{q}(g)}{g} \leq \sqrt{q}-1
$$

\section{Preliminaries}

Let $X$ be a complete, geometrically irreducible, algebraic curve defined over a finite field $k=\mathbb{F}_{q}$ with $q$ elements. We denote by $\pi: \widetilde{X} \longrightarrow X$ the morphism of normalization of $X$, by $g$ its arithmetical genus and by $\tilde{g}$ its geometric genus. The curve $X \times_{k} \bar{k}$ is denoted by $\bar{X}$, where $\bar{k}$ is the algebraic closure of $k$.

From now on, we use point to mean closed point. Since the function fields of $X$ and $\widetilde{X}$ are the same, we denote this function field by $K / k$. Let $P$ be a point of $X$. We denote by $\widetilde{\mathcal{O}}_{P}$ the integral closure of the local ring $\mathcal{O}_{P}$ of $X$ at $P$ in its quotient field, i.e. in $K / k$,

$$
\widetilde{\mathcal{O}}_{P}=\bigcap_{Q \in \pi^{-1}(P)} \mathcal{O}_{Q}
$$

where $\mathcal{O}_{Q}$ is the local ring of $\tilde{X}$ at $Q$. Since $\tilde{X}$ is a non-singular curve, $\mathcal{O}_{Q}$ is a valuation ring of $K / k$. Thus the local properties of an algebraic curve can be studied in terms of local rings of its function field, i.e., without explicit reference to the curve.

Let $K / k$ be a function field with constant field $k$ and $\mathcal{O}$ a local ring of $K$. We denote by $\tilde{\mathcal{O}}$ its integral closure in $K / k$. The degree of singularity of $\mathcal{O}$ is defined as

$$
\delta=\operatorname{dim}_{k}(\tilde{\mathcal{O}} / \mathcal{O})
$$


By thm. 1 of [Ros1] we have that $\delta<\infty$. $\delta$ remains invariant under completion with respect to the topology induced by the maximal ideal of $\mathcal{O}$ and under separable constant extensions (cf. [Ros1], thm. 12). Alternatively, if $k^{\prime} / k$ is a separable extension, the integral closure of $\mathcal{O} \otimes_{k} k^{\prime}$ is isomorphic to $\tilde{\mathcal{O}} \otimes_{k} k^{\prime}$. We denote by $\mathcal{F}$ the conductor ideal of $\tilde{\mathcal{O}}$ in $\mathcal{O}$, i.e.,

$$
\mathcal{F}=\{x \in K \mid x \tilde{\mathcal{O}} \subseteq \mathcal{O}\} .
$$

Since $\tilde{\mathcal{O}}$ is a Dedekind domain with a finite number of maximal ideals, $\mathcal{F}$ is an $\tilde{\mathcal{O}}$-principal ideal.

In the case in which $\mathcal{O}$ corresponds to the local ring of an algebraic curve at $P$, we denote the degree of singularity of $\mathcal{O}$ by $\delta_{P}$, and call it the local degree of singularity at $P$. We recall the Hironaka genus formula for an algebraic curve (cf. $[\mathrm{H}])$

$$
g=\tilde{g}+\sum_{P \in X} \delta_{P}
$$

The following result will be used in the next section.

Theorem 2.1 (cf. [Ros2, thm. 11]). Let $K / k$ be a function field, where the constant field $k$ is an algebraically closed field. Let $\mathcal{O}$ be a local ring of $K / k$. Then the quotient group $J(\mathcal{O}, k)=\tilde{\mathcal{O}}^{*} / \mathcal{O}^{*}$ is an affine, irreducible, commutative, algebraic group of dimension $\delta$. More precisely,

$$
J(\mathcal{O}, k) \cong G_{m}^{d-1} \times \Gamma(\mathcal{O}),
$$

where $G_{m}$ is the algebraic multiplicative group of $k, d$ is the number of valuation rings dominating $\mathcal{O}$ and $\Gamma(\mathcal{O})$ is an algebraic group. Thus we have $\delta=d-1+\gamma$, where $\gamma$ is the dimension of the group $\Gamma$ in (2.2).

The structure of the group $\Gamma(\mathcal{O})$ is well known if $X$ is obtained from a modulus (cf. [Ser3], Chap. IV). In this case $\Gamma(\mathcal{O})$ is a product of groups of type $U^{(1)} / U^{(n)}$, where $U^{(n)}=\left\{z \in k[[t]] \mid z=1+a_{n} t^{n}+\right.$ higher order terms, $\left.a_{n} \in k^{*}\right\}$, for some $n$. $U^{(1)} / U^{(n)}$ is an irreducible, affine, algebraic group of dimension n (cf. [Ser3], chap. $\mathrm{V}$, sect. 15, 16). In zero characteristic, the group $U^{(1)} / U^{(n)}$ is a product of groups of the form $G_{a}$, where $G_{a}$ denotes the additive algebraic group of $k$. In positive characteristic, $U^{(1)} / U^{(n)}$ is a product of groups of Witt vectors of finite length. In the general case $\Gamma(\mathcal{O})$ is product of subgroups of $U^{(1)} / U^{(n)}$, for some $n$ 's. The groups $\tilde{\mathcal{O}}_{P}^{*} / \mathcal{O}_{P}^{*}$ appear naturally in the study of the generalized jacobian of $X$ (cf. [Ros2], thm. 11).

Let $X$ be a complete, geometrically irreducible, algebraic curve defined over a finite field $k$. We define

$$
\delta:=\sum_{P \in \bar{X}_{\text {sing }}} \delta_{P}
$$

where $\delta_{P}$ is the local degree of singularity at $\mathrm{P}$, and

$$
\gamma:=\sum_{P \in \bar{X}_{\text {sing }}} \gamma_{P}
$$

where $\gamma_{P}$ is the dimension of the algebraic group $\Gamma\left(\mathcal{O}_{P}\right)$ in the product $(2.2)$. 
Let $X$ be a complete, geometrically irreducible, algebraic curve defined over a finite field $k=\mathbb{F}_{q}$. Its zeta function is defined by

$$
\zeta(X, t)=\prod_{P \in X}\left(1-t^{\operatorname{deg}(P)}\right)^{-1}, \text { where } t=q^{-s}, \quad s \in \mathbb{C}, \quad \operatorname{Re}(s)>1,
$$

and $\operatorname{deg}(P)=\left[\mathcal{O}_{P} / \mathcal{M}_{P}: k\right]$, where $\mathcal{M}_{P}$ denotes the maximal ideal of $\mathcal{O}_{P}$. This zeta function contains diophantine information about $X$ [Ser1], i.e.,

$$
\zeta(X, t)=\exp \left(\sum_{n \geq 1} \frac{\# X\left(\mathbb{F}_{q^{n}}\right)}{n} t^{n}\right),
$$

where $\# X\left(\mathbb{F}_{q^{n}}\right)=\sum_{\{P|\operatorname{deg}(P)| n\}} \operatorname{deg}(P)$ is the number of $\mathbb{F}_{q^{n}}$-rational points of $X$.

\section{Bounding the NUMBer OF POINTS ON A SINGULAR CURVE}

In this section we prove the estimation (1.4) for the number of rational points of a complete, geometrically irreducible, algebraic curve.

Theorem 3.1. Let $X$ be a complete, geometrically irreducible, algebraic curve defined over a finite field $k=\mathbb{F}_{q}$. Then the number of rational points on $\mathbb{F}_{q^{n}}$ satisfies

$$
\left|\# X\left(\mathbb{F}_{q^{n}}\right)-\left(q^{n}+1\right)\right| \leq 2 \tilde{g} q^{\frac{n}{2}}+\delta-\gamma .
$$

Proof. We recall that

$$
\zeta(X, t)=\exp \left(\sum_{n \geqq 1} \frac{\# X\left(\mathbb{F}_{q^{n}}\right)}{n} t^{n}\right),
$$

where $\# X\left(\mathbb{F}_{q^{n}}\right)$ is the number of $\mathbb{F}_{q^{n} \text {-rational points of } X \text {. Therefore }}$

$$
\zeta(X, t)=\zeta(\widetilde{X}, t) M(X, t)
$$

where

$$
M(X, t):=\prod_{P \in X_{\text {sing }}} \frac{\prod_{Q \in \pi^{-1}(P)}\left(1-t^{\operatorname{deg}(Q)}\right)}{1-t^{\operatorname{deg}(P)}} .
$$

$M(X, t)$ is a polynomial in $\mathbb{Z}[t]$ whose roots lie on the unit circle.

Taking logarithmic derivatives in (3.2) and multiplying by $t$, we get

$$
\begin{gathered}
\sum_{n \geqq 1} \# X\left(\mathbb{F}_{q^{n}}\right) t^{n}-\sum_{n \geqq 1} \# \widetilde{X}\left(\mathbb{F}_{q^{n}}\right) t^{n} \\
=\sum_{P \in X_{\text {sing }}}\left(\sum_{Q \in \pi^{-1}(P)} \frac{-\operatorname{deg}(Q) t^{\operatorname{deg}(Q)}}{1-t^{\operatorname{deg}(Q)}}+\frac{\operatorname{deg}(P) t^{\operatorname{deg}(P)}}{1-t^{\operatorname{deg}(P)}}\right) .
\end{gathered}
$$

We define

$$
\chi(a, n):=\left\{\begin{array}{l}
1 \text { if } n \mid a, \\
0 \text { if } n \nmid a .
\end{array}\right.
$$

Using the fact that

$$
\frac{t^{s}}{1-t^{s}}=t^{s}+t^{2 s}+t^{3 s}+\ldots
$$


and counting how many times $n$ appears as a power on the right-hand side, we conclude from (3.3) that

$$
\# \widetilde{X}\left(\mathbb{F}_{q^{n}}\right)=\# X\left(\mathbb{F}_{q^{n}}\right)+\sum_{P \in X_{\text {sing }}} \Delta_{P}^{(n)},
$$

where $\Delta_{P}^{(n)}$ is given by

$$
\left.\Delta_{P}^{(n)}=\sum_{Q \in \pi^{-1}(P)} \operatorname{deg}(Q) \chi(\operatorname{deg}(Q), n)\right)-\operatorname{deg}(P) \chi(\operatorname{deg}(P), n) .
$$

We claim that

$$
\sum_{P \in X_{\text {sing }}}\left|\Delta_{P}^{(n)}\right| \leq \delta-\gamma
$$

The result follows from (3.4), the above inequality, and Weil's bound applied to $\tilde{X}\left(\mathbb{F}_{q^{n}}\right)$. To complete the proof we prove (3.6).

First, we note that $\operatorname{deg}(P) \mid \operatorname{deg}(Q)$ for all $Q \in \pi^{-1}(P)$. If $\operatorname{deg}(P) \nmid n$, we have $\Delta_{P}^{(n)}=0$. If $\operatorname{deg}(P) \mid n$, we consider two cases. The first occurs when $\operatorname{deg}(Q) \nmid$ $n$ for all $Q \in \pi^{-1}(P)$. In this case $\Delta_{P}^{(n)}=-\operatorname{deg}(P)$. The second one occurs when $\operatorname{deg}(Q) \mid n$, for $Q \in S, S \subseteq \pi^{-1}(P)$. In this case, we have

$$
\Delta_{P}^{(n)}=\sum_{Q \in S} \operatorname{deg}(Q)-\operatorname{deg}(P) .
$$

Summarizing, the possible values of $\Delta_{P}^{(n)}$ are:

(1) 0 if $\operatorname{deg}(P) \nmid n$,

(2) 0 if $\# \pi^{-1}(P)=1, \operatorname{deg}(P)=\operatorname{deg}(Q)$,

(3) $-\operatorname{deg}(P)$ if $\operatorname{deg}(P) \mid n, \quad \operatorname{deg}(Q) \nmid n$, for each $Q \in \pi^{-1}(P)$,

(4) $\sum_{Q \in S} \operatorname{deg}(Q)-\operatorname{deg}(P)$, where $S=\left\{Q \in \pi^{-1}(P)|\operatorname{deg}(Q)| n\right\}$.

From the above list, we conclude that the maximum value of $\left|\Delta_{P}^{(n)}\right|$ is

$$
\sum_{Q \in \pi^{-1}(P)} \operatorname{deg}(Q)-\operatorname{deg}(P)=\operatorname{deg}(P)\left(-1+\sum_{Q \in \pi^{-1}(P)} \frac{\operatorname{deg}(Q)}{\operatorname{deg}(P)}\right),
$$

a nonnegative integer multiple of $\operatorname{deg}(\mathrm{P})$. We recall that the closed points of $X$ correspond bijectively to orbits of points of $\bar{X}$ under the action of the group $\operatorname{Gal}\left(\overline{\mathbb{F}_{q}} / \mathbb{F}_{q}\right)$. Furthermore, the corresponding orbit of a closed point $P$ has $\operatorname{deg}(P)$ points. We denote the orbit corresponding to $P$ by $\operatorname{Conj}(P)$. Let $P$ be a singular point of $X$. By theorem 2.1 (the number $d$ can be computed as $\left.\sum_{Q} \frac{\operatorname{deg}(Q)}{\operatorname{deg}(P)}\right)$, for each $P^{\prime} \in$ $\operatorname{Conj}(P)$, we have

$$
\delta_{P^{\prime}}=\frac{1}{\operatorname{deg}(P)} \sum_{Q \in \pi^{-1}(P)} \operatorname{deg}(Q)+\gamma P^{\prime}-1 .
$$

Therefore

$$
\sum_{Q \in \pi^{-1}(P)} \operatorname{deg}(Q)-\operatorname{deg}(P)=\operatorname{deg}(P)\left(\delta_{P^{\prime}}-\gamma_{P^{\prime}}\right)=\sum_{P^{\prime} \in \operatorname{Conj}(P)}\left(\delta_{P^{\prime}}-\gamma_{P^{\prime}}\right) .
$$

Finally, from (3.7) and (3.9), we get

$$
\sum_{P \in X_{\text {sing }}}\left|\Delta_{P}^{(n)}\right| \leq \sum_{P \in X_{\text {sing }}} \sum_{P^{\prime} \in \operatorname{Conj}(P)}\left(\delta_{P^{\prime}}-\gamma_{P^{\prime}}\right)=\delta-\gamma .
$$


Example 3.2. Let $\widetilde{X}$ be a complete, geometrically irreducible, non-singular, algebraic curve, with function field $K / \mathbb{F}_{q}$. Let $S=\left\{Q_{1}, Q_{2}, \ldots, Q_{d}\right\}$ be $d$ rational points of $\widetilde{X}$ with $d \geqq 2$. Let $n_{Q_{1}}, n_{Q_{2}}, . ., n_{Q_{d}}, d$ be arbitrary positive integers. We consider the local ring $\mathcal{O}_{P_{0}}=k+\mathcal{F}_{P_{0}}$ of $K / \mathbb{F}_{q}$, where $\mathcal{F}_{P_{0}}$ is given by

$$
\mathcal{F}_{P_{0}}=\left\{x \in K \mid v_{Q}(x) \geq n_{Q}, Q \in S\right\},
$$

where $v_{Q}$ is the valuation associated to $\mathcal{O}_{Q}$. There exists a complete, geometrically irreducible, algebraic curve $X$ with function field $K / \mathbb{F}_{q}$ having only a singular point at $P_{0}$, with local ring isomorphic to $\mathcal{O}_{P_{0}}$ (cf. [Ros1], thm. 5).

Let $M=\prod_{Q \in \tilde{X}} \mathcal{M}_{Q}^{n_{Q}}$ be a positive divisor with support $\mathrm{S}$. The curve $X$ has been obtained from $\tilde{X}$ and the modulus $M$ by identifying all points in the support of $M$ with the point $P_{0}$ (cf. [Ser3], chap. IV). By [Ser3] (chap. V, sect. 17), the group $\Gamma\left(\mathcal{O}_{P_{0}} \bigotimes_{k} \bar{k}\right) \cong G_{m}^{d-1} \times \prod_{Q \in S} U^{(1)} / U^{\left(n_{Q}-1\right)}$. Therefore $\gamma=\sum_{Q \in S}\left(n_{Q}-1\right), \delta=$ $-1+\sum_{Q \in S} n_{Q}$. Fixing $d$ and considering the $n_{Q}$ 's as arbitrary, we see that $\delta, \gamma$ can be arbitrarily large, but the difference $\delta-\gamma$ is constant. Thus $\left|\# \tilde{X}\left(\mathbb{F}_{q}\right)-\# X\left(\mathbb{F}_{q}\right)\right|=$ $d-1=\delta-\gamma$, and the bound (1.4) is attained. Since the bound (1.3) depends only on $\delta$, it is too bad. More precisely, $\left|\# \tilde{X}\left(\mathbb{F}_{q}\right)-\# X\left(\mathbb{F}_{q}\right)\right|=d-1 \leq-1+\sum_{Q \in S} n_{Q}$.

Example 3.3. Let $\widetilde{X}$ be a complete, geometrically irreducible, non-singular, algebraic curve, with function field $K / \mathbb{F}_{q}$. Let $Q_{0}$ be a point of $\widetilde{X}$ of degree $n \geq 2$. We consider the local ring $\mathcal{O}_{Q_{0}}=\mathbb{F}_{q}+\mathcal{F}_{Q_{0}}$ of $K / \mathbb{F}_{q}$, where $\mathcal{F}_{P_{0}}=\left\{x \in K \mid v_{Q_{0}}(x) \geq 0\right\}$ and $v_{Q_{0}}$ is the valuation associated to $\mathcal{O}_{Q_{0}}$. Then there exists a complete, geometrically irreducible, algebraic curve $X$ that has only a singular point at $P_{0}$ with local ring isomorphic to $\mathcal{O}_{P_{0}}$. The modulus $M$ is a positive divisor with support equal to $\left\{Q_{0}\right\}$. In order to compute $\gamma_{P_{0}}$, we take an extension of degree $n$ of the base field $\mathbb{F}_{q}$. From lemma 4 of $[\mathrm{H}]$, we see that $\mathcal{F}_{P_{0}} \otimes_{\mathcal{F}_{q}} \mathbb{F}_{q^{n}}$ is the conductor of $\widetilde{\mathcal{O}} \otimes_{\mathbb{F}_{q}} \mathbb{F}_{q^{n}}$ in $\mathcal{O}_{P_{0}} \otimes_{\mathbb{F}_{q}} \mathbb{F}_{q^{n}}$. Thus $\mathcal{O}_{P_{0}} \otimes_{\mathbb{F}_{q}} \mathbb{F}_{q^{n}}=\mathbb{F}_{q^{n}}+\mathcal{F}_{P_{0}} \otimes_{\mathbb{F}_{q}} \mathbb{F}_{q^{n}}$, where $\mathcal{F}_{P_{0}} \otimes_{\mathbb{F}_{q}} \mathbb{F}_{q^{n}}=\left\{x \in K \mid v_{Q}(x) \geq 0\right\}$, with $\mathrm{Q}$ running through all valuation rings of $K \otimes_{\mathbb{F}_{q}} \mathbb{F}_{q^{n}}$ lying above $\mathcal{O}_{P_{0}}$. Now we have $\Gamma\left(\mathcal{O}_{P_{0}} \bigotimes_{k} \bar{k}\right) \cong G_{m}^{n-1}$ (cf. [Ser3], chap. $\mathrm{V}$, sect. 17). Thus $\delta=n-1, \gamma_{P_{0}}=0$. In this case, the bounds 1.3 and 1.4 give $\left|\# \tilde{X}\left(\mathbb{F}_{q}\right)-\# X\left(\mathbb{F}_{q}\right)\right|=1 \leq n-1$.

\section{Asymptotic Bounds on the number of Points on CURVES}

The following result generalizes the Drinfeld-Vladut asymptotic bound:

\section{Theorem 4.1.}

$$
\mathcal{A}(q)=\limsup _{g \rightarrow \infty} \frac{N_{q}(g)}{g} \leq \sqrt{q}-1 .
$$

The following technical result is used in the proof of the above theorem. We set

$$
\Delta_{n}:=\# \widetilde{X}_{n}\left(\mathbb{F}_{q}\right)-\# X_{n}\left(\mathbb{F}_{q}\right) .
$$

By (3.4), $\Delta_{n}=\sum_{P \in X_{n_{s i n g}}} \Delta_{P}^{(1)}$ (cf. 3.5).

Lemma 4.2. Let $\left(X_{n}, g_{n}\right)$ be a sequence of complete, geometrically irreducible, singular curves defined over $\mathbb{F}_{q}$, such that the geometric genus of each $X_{n}$ is constant, $\tilde{g}_{n}=\tilde{g}_{0}$, and $\delta_{n}$ goes to infinity. Then

$$
\lim _{n \rightarrow \infty} \frac{\Delta_{n}}{\delta_{n}}=0 .
$$


Proof. If $\Delta_{n}$ is bounded, the result is true. Thus we can suppose that $\Delta_{n} \longrightarrow \infty$. We define

$$
B_{n}\left(\mathbb{F}_{q}\right)=\left\{P \in X_{n_{\text {sing }}} \mid \text { there exists } Q \in \pi_{n}^{-1}(P) \text { with } \operatorname{deg}(Q)=1\right\} .
$$

Since $\Delta_{n}>0$ if $n>>0$, by (3.5), we get

$$
\Delta_{n}=\sum_{P \in B_{n}\left(\mathbb{F}_{q}\right)}\left(\left(\sum_{Q \in \pi_{n}^{-1}(P)} 1\right)-1\right) .
$$

We consider two cases. The first one occurs when $\# B_{n}\left(\mathbb{F}_{q}\right)$ is a bounded sequence. In this case, the condition $\Delta_{n} \longrightarrow \infty$ implies that there exists at least one point $P_{n} \in B_{n}\left(\mathbb{F}_{q}\right)$ such that

$$
\#\left\{Q \in \pi_{n}^{-1}\left(P_{n}\right) \mid \operatorname{deg}(Q)=1\right\} \longrightarrow \infty .
$$

In either of the two cases, we have $\# \widetilde{X}_{n}\left(\mathbb{F}_{q}\right) \longrightarrow \infty$. Weil's bound implies that $\tilde{g}_{n} \longrightarrow \infty$ ( $q$ is fixed). But this contradicts the initial hypothesis that the geometric genus of all $\widetilde{X}_{n}$ is constant. Thus $\Delta_{n}$ is always bounded, and (4.1) is true.

Proof of theorem 4.1. If the theorem is false, let $A(q)>\sqrt{q}-1$. In this situation, there exists a sequence of singular curves $\left(X_{n}, g_{n}\right)$ defined over $\mathbb{F}_{q}$, such that the sequence $\frac{\# X_{n}\left(\mathbb{F}_{q}\right)}{g_{n}}$ is convergent and

$$
\lim _{g_{n} \rightarrow \infty} \frac{\# X_{n}\left(\mathbb{F}_{q}\right)}{g_{n}}>\sqrt{q}-1 .
$$

Therefore, $\frac{\# X_{n}\left(\mathbb{F}_{q}\right)}{g_{n}}>\sqrt{q}-1$ if $n>>0$. We denote by $\tilde{X}_{n}$ the normalization of $X_{n}$ over $k$, by $\pi_{n}: \tilde{X}_{n} \longrightarrow X_{n}$ the normalization morphism of $X_{n}$, and $\tilde{g}_{n}$ denotes the geometric genus of $X_{n}$. The Hironaka genus formula implies $\tilde{g}_{n}=g_{n}-\delta_{n}$, where $\delta_{n}$ is the total degree of singularity of $X_{n}$.

We consider two cases. The first one occurs when the sequence $\tilde{g}_{n}$ is bounded. In this case, Weil's bound and hypothesis (4.2) imply

$$
\sqrt{q}-1<\lim _{n \rightarrow \infty} \frac{\# X_{n}\left(\mathbb{F}_{q}\right)}{g_{n}}=\lim _{n \rightarrow \infty} \frac{\# \widetilde{X}_{n}\left(\mathbb{F}_{q}\right)-\Delta_{n}}{\widetilde{g}_{n}+\delta_{n}}=\lim _{n \rightarrow \infty}-\frac{\Delta_{n}}{\delta_{n}} .
$$

From (4.3) and Lemma 4.2, we obtain

$$
\sqrt{q}-1<0 .
$$

Thus this case cannot occur. In the other case, where $\tilde{g}_{n}$ is not bounded, i.e., $g_{n} \longrightarrow \infty$, hypothesis (4.2) implies

$$
\# X_{n}\left(\mathbb{F}_{q}\right)-\tilde{g}_{n}(\sqrt{q}-1)>\Delta_{n}+\delta_{n}(\sqrt{q}-1),
$$

if $n \gg 0$. The Drinfeld-Vladut bound for non-singular curves (cf. [D-V]) implies that

$$
\# \widetilde{X}_{n}\left(\mathbb{F}_{q}\right)-\tilde{g}_{n}(\sqrt{q}-1) \leqq 0,
$$

if $\tilde{g}_{n} \gg 0$. Thus from (4.5) and (4.4), we get

$$
(\sqrt{q}-1) \delta_{n}+\Delta_{n}<0,
$$

if $n \gg 0$. Since $\Delta_{n} \geqq 0$ and $\delta_{n} \geqq 0$, this case cannot occur. Thus, the result is true. 
Corollary 4.3. Let $\left(X_{n}, g_{n}\right)$ be a sequence of complete, geometrically irreducible, algebraic curves defined over $\mathbb{F}_{q}$, attaining the Drinfeld-Vladut bound. If the sequence $\delta_{n}$ is bounded, then the sequence of non-singular curves $\left(\widetilde{X}_{n}, \tilde{g}_{n}\right)$ also attains the Drinfeld-Vladut bound.

Proof. The result follows from the above reasoning.

\section{REFERENCES}

[A-P] Aubry, Y., Perret, M., A Weil theorem for singular curves., proceedings of Arithmetic, Geometry and Coding Theory IV, Ed. Pellikaan, Perret, Vladut, De Gruyter, (1995). MR 97g:11061

[D-V] Drinfeld, V. and Vladut, S., Number of points of an algebraic curve, Func. Anal. Appl., 17, 53-54, (1983). MR 85b:14028

[H] Hironaka, H., On the arithmetic genera and effective genera of algebraic curves, Mem. Kyoto, 30,177-195, (1957). MR 19:881b

[Ros1] Rosenlicht, M., equivalence relations on algebraic curves, Ann. of Math. ,56,169-191, (1952). MR 14:80c

[Ros2] Rosenlicht, M., Generalized jacobian varieties, Ann. of Math. ,59,503-530, (1954). MR 15: $823 \mathrm{~b}$

[Ser1] Serre, J.P., Zeta and L functions, Arithmetical algebraic geometry, Harper-Row, N-Y., 82-92, (1965). MR 33:2606

[Ser2] Serre, J.P., Rational points on curves over finite fields, notes by Fernando Q. Gôuvea, unpublished.

[Ser3] Serre, J.P., Algebraic groups and class fields, Springer-Verlag, (1988). MR 88i:14041

[Sto] Stöhr, K.O., On poles of regular differentials of singular curves, Bol. Soc. Bras. Mat., 24,105-136, (1993). MR 94e:14035

Instituto de Matemática Pura e Aplicada, Estrada Dona Castorina 110, CEP 22460320, Rio de Janeiro-R.J., BraziL

Current address: Universidad Autónoma de Bucaramanga, Laboratorio de Computo Especializado, A.A.1642, Bucaramanga, Colombia

E-mail address: wzuniga@bumanga.unab.edu.co 